\title{
Comments on "Tactus $\neq$ Tempo: Some Dissociations Between Attentional Focus, Motor Behavior, and Tempo Judgment" by Justin London
}

\author{
BRUNO H. REPP \\ Haskins Laboratories
}

\begin{abstract}
Objective measures of musical tempo are linked to a particular metrical pulse, and this is most likely true for subjective tempo as well. Therefore, tapping along with a rhythm should be predictive of relative tempo judgments. The difficulty of such judgments may lie in the choice of a particular metrical level as the referent. Multiple levels may compete and create ambiguity. Analogies are drawn with pitch perception from certain types of complex tones.
\end{abstract}

Submitted 2011 January 19; accepted 2011 February 14.

KEYWORDS: tempo, meter, tactus, tapping, pitch, timbre

LONDON (2011) has made an interesting empirical contribution to the study of tempo judgment. He asked participants to compare the tempi of simple isochronous rhythms in which one or two higher metrical levels were highlighted by regularly recurring pitch accents. When two different rhythmic patterns were presented in succession, participants often did not make their judgments according to the tempo of the nominal tactus (the quarter note). London's main conclusion is that "our perception of musical speed or tempo is more than simple apprehension of the tactus rate" (p. 1). Does he mean the nominal tactus rate or any tactus rate?

\section{NOMINAL VERSUS SUBJECTIVE TACTUS}

The rate of the nominal tactus of his materials ( 100 or 120 beats per minute) was chosen to be in the most preferred region for a musical beat (Parncutt, 1994; van Noorden \& Moelants, 1999). However, that does not guarantee that participants would always choose the nominal tactus as the most salient metrical level. The subjective tactus might differ, especially when higher metrical levels carry regular pitch accents. Indeed, in his Experiment 3, where participants were required to tap along with each rhythm, London found that they did not always tap with the nominal tactus. Presumably, they tapped with their subjective tactus.

One reasonable conjecture then is that participants made their tempo judgments according to their subjective tactus, regardless of whether they did or did not tap along with it. London found that instructions to "focus on the beat rate" (p. 3, Experiment 2) or "tap along with the beat" (p. 4, Experiment 3) had relatively little effect on participants' tempo judgments. From his description it seems he did not instruct participants to focus on or tap along with the quarter-note beat, only with "the beat." That beat then was most likely the subjective tactus, and if it already formed the basis of tempo judgments in Experiment 1, then it is understandable that focusing on or tapping along with it had little additional effect.

If my conjecture is right, then the tempo judgments in Experiment 3 should have been consistent with the tapping tempi. Although London examined these tempi and related them to different metrical levels, he does not report how they related to the tempo judgments. If participants tapped faster to one rhythm than to another on any given trial, did they always (or nearly always) judge the tempo of the former to be faster than the tempo of the latter? This question could easily be addressed by further analysis of the data of Experiment 3. If the answer is positive, it would suggest that the subjective tempo of a rhythm is simply the tempo of the subjective tactus, and London's conclusion that "tactus $\neq$ tempo" (the title of his paper) might not be justified. 
However, this may not be the whole story. Quite possibly, participants sometimes tapped to two rhythms at the same rate but nevertheless judged the tempi to be different, or tapped at different rates and judged the tempi to be the same. (It would be good to see whether and how often that was actually the case.) If this occurred, how should such responses be explained? One possibility is that participants did not always tap with their subjective tactus in Experiment 3, although they relied on it in their tempo judgments. That seems unlikely given that they had been encouraged to tap with the beat of the music.

\section{IS THERE A “MUSICAL SPEED”?}

Another possibility that seems to be favored by London is that subjective tempo is not linked to a particular metrical level but rather is a continuously variable quantity. London quotes Epstein (1995), according to whom "tempo is the sum of all factors within a piece" (p. 2). Epstein, however, was concerned with finding the best tempo at which to perform a musical piece - the tempo that seems subjectively right for it given all its structural properties. (See Quinn \& Watt, 2006, for a recent empirical study along these lines.) In London's study, participants had to compare the given tempi of two rhythms, which is quite a different task.

London asks right at the outset whether metronome markings "really give an accurate indication of our subjective sense of musical speed" (p. 1), implying that they do not. Indeed, metronome markings cannot give such an indication. They convey the suggested rate of one particular pulse of the music, usually (though not always) the nominal tactus. A metronome marking must be preceded by a note symbol that refers to a particular metrical level; without that information, the number indicating beats per minute would be meaningless. Even an optimal tempo such as envisioned by Epstein is still described in beats per minute - that is, with reference to a particular metrical level. ${ }^{[1]}$

Any measure of rate or speed, whether objective or subjective, must refer to something that occurs at that rate or moves at that speed. In music, this something is a more or less isochronous pulse at some level of the metrical hierarchy. Music that does not have any such pulse also (arguably) has no tempo. ${ }^{[2]}$ What makes music "move," in my view, is its rhythm, which can be decomposed into metrical levels. Therefore, I believe the notion of a subjective musical speed that is divorced from metrical structure is a misconception.

However, because metrical music has at least two, often three or four, hierarchical levels, each of which typically corresponds to an isochronous pulse with a certain tempo, each piece of music really has a multiplicity of possible tempi. This can lead to uncertainty as to which level is to constitute the reference level, and consequently to ambiguity regarding tempo or outright plurality of perceived tempi.

\section{AN ANALOGY WITH PITCH PERCEPTION IN SHEPARD TONES}

An analogy with pitch perception might be instructive here. Rhythm and complex harmonic sounds have some structural similarities, despite the obvious difference in time scale. A periodic sound will be perceived as a rhythm if its frequency is $2 \mathrm{~Hz}$, but as a pitch if its frequency is $200 \mathrm{~Hz}$. The type of sound most analogous to rhythms with a binary metrical hierarchy, as used in London's study, is the Shepard tone (Shepard, 1964). A Shepard tone is composed of a number of partials, with adjacent partials being separated by the interval of an octave, so that their frequency ratios are 1:2:4:8.. The perceived pitch of such a tone is known to be ambiguous with regard to height, though not with regard to chroma (pitch class). For example, a tone composed of partials with frequencies of $110,220,440$, and $880 \mathrm{~Hz}$ will always be perceived as an A natural, but that A may be A2, A3, A4, or A5. In other words, the perceived pitch always corresponds to one of the partials, never to anything in between. There may be uncertainty about the dominant pitch, and indeed listeners often report hearing multiple pitches simultaneously. However, just as often one of the partials will be perceptually more salient than the others and determine "the" perceived pitch of the complex tone. Interestingly, unlike the "fundamental frequency" of ordinary harmonic tones, that dominant partial is not usually the lowest one in the spectrum. Rather, partials near $300 \mathrm{~Hz}$ seem to be favored perceptually (Repp \& Thompson, 2010; Terhardt et al., 1986). 
Let us now extend the analogy backward to rhythm. The metrical levels of a binary metrical hierarchy have interval ratios of 1:2:4:8. The perceived tempo corresponds (I claim) to one or several of these levels, not to something in between. The tactus is the most salient "partial" of a metrical hierarchy and thus represents the "pitch" (i.e., tempo) of a rhythmic complex. It is well known that there is a (broad) preference region for the tactus, centered on $2 \mathrm{~Hz}$ or a little lower (Parncutt, 1994; van Noorden \& Moelants, 1999). However, other "partials" can become equally or even more salient, especially if they are highlighted through accentuation, in which case they can become the tactus and determine the perceived tempo or at least create uncertainty about which of several pulses is the tactus, and hence which of several possible tempi the rhythm has.

Madison (2009) has provided an elegant demonstration of the structural and perceptual analogy between the pitch of Shepard tones and the tempo of hierarchical metrical rhythms. He presented participants with a continuously changing rhythm that created an auditory illusion of steadily increasing or decreasing tempo, which participants indicated by tapping along. This illusion is analogous to Shepard's (1964) "scale illusion", in which a chromatically ascending or descending sequence of Shepard tones is perceived as continuously increasing or decreasing in pitch. Shepard tones have a fixed (typically bellshaped) spectral amplitude envelope, so that in an ascending sequence, for example, a new low partial emerges as the highest partial fades away, with this process repeating itself every 12 tones in the sequence. When listening to Shepard scales, many listeners never experience abrupt shifts in pitch; rather, their (ambiguous or multivalent) pitch percept shifts gradually between adjacent partials in the direction contrary to that of the scale, so that they never hear implausibly high or low pitches. In Madison's experiment, participants could not shift smoothly between pulse levels because they were tapping in synchrony with one of them, but they were remarkably tenacious in holding on to that level before abruptly shifting to a lower or higher level.

What are the implications of these observations for London's experiments? My conjecture is that his participants, rather than gaining a vaguely defined "sense of musical speed" (p. 2) from the presented rhythmic patterns, were either focusing on one metrical level (their subjective tactus) or, in the case of two equally salient levels, were confused about which level they should choose for their tempo judgment (and hence vacillated from trial to trial or even within a trial). London only requested "slower", "same", and "faster" judgments, and did not obtain a measure of how much slower or faster participants thought one rhythm was compared to another. Quantitative judgments of the tempo difference might prove informative about the basis of the judgment. For example, if two rhythms are presented at the same nominal tactus tempo but are judged to differ in tempo, is one of the rhythms perceived as being twice as fast than the other or just as being a little faster? If twice as fast, this would be very consistent with my hypothesis that the relative tempo judgment is based on different subjective tactus choices for the two rhythms. If just a little faster, this would suggest that the same tactus was chosen, but that its tempo was slightly misperceived in one or both rhythms.

\section{MISPERCEPTION OF TEMPO}

Subjective tempo does not have to match the objective tactus rate exactly, just as the perceived pitch of a tone need not match exactly the fundamental frequency of a tone. The occurrence of such small deviations reveals that tempo and pitch are subjective quantities, but they are nevertheless closely tied to the physical stimulus. For example, I have shown in recent research (Repp, 2008; Repp \& Bruttomesso, 2009) that an explicit beat (marked by tone onsets) is perceived as slightly slower when it is explicitly subdivided by additional tones than when the inter-beat intervals are empty. This seems contrary to what London found. In his Table 5, he shows that the majority of "wrong" responses to pairs of different rhythms played at different nominal tactus tempi "corresponded to the surface rates of motion" (p. 9). In other words, participants often judged that rhythm to be faster which had the fastest explicit pulse. Thus, for example, when rhythms 1 and 2 (see London's Figure 2) were to be compared, participants would often judge rhythm 1 to be faster, even when its quarter-note pulse was slower than that of rhythm 2 .

Suppose London had instructed his participants to compare not the tempi of the rhythms but specifically the tempi of their quarter-note pulses (their nominal tactus). My contention is that, in that case, they would have judged rhythm 2 as faster because its nominal tactus is not subdivided. As far as I can see, London did not present such instructions but rather left it up to the participants to choose their own tactus. 
Nevertheless, he classified responses as "wrong" or not according to the relative tempi of the nominal tactus of the two rhythms. His use of scare quotes indicates that he is aware that this classification is somewhat arbitrary. Since the nominal tactus was not pointed out to the participants, it had little reality for them apart from a weak preference for a subjective tactus having a similar rate.

The "wrong" responses identified by London could have two sources. One is that participants chose a faster tactus when the surface rate of motion was faster. Thus they may have focused on the quarter-note level in rhythm 1, but on the half-note level in rhythm 2. In that connection, it might be noted that some rhythms (e.g., 5, 1, and 7) resembled other rhythms $(3,2$, and 5, respectively) being played twice as fast. This certainly could be a source of confusion. Indeed, at the same nominal tactus tempo a sequence of eighth notes is objectively twice as fast as a series of quarter notes, and to call it faster is not a "wrong" response. It is so only from the perspective of a predefined quarter-note tactus.

\section{AN ANALOGY WITH PITCH AND TIMBRE PERCEPTION}

The other potential source of "wrong" responses can be illustrated once again with an analogy to pitch perception, now including timbre. The timbre of a complex tone is determined primarily by its frequency spectrum: the more high-frequency components there are in a sound, the brighter its timbre will seem, even though its pitch may be the same. Like pitch, timbre depends on frequency components that range from low to high, and the dull-bright timbre continuum therefore is similar to the low-high pitch continuum. In pure tones, pitch and timbre are co-determined by frequency: a higher tone is necessarily also brighter (although this fact is rarely pointed out). A low complex tone can have a bright timbre if its higher harmonics are strong, but a high complex tone cannot have a dull timbre because low harmonics are lacking. Pitch and timbre are thus not independent, and listeners who are not musically trained often confuse them (Pitt, 1994; Pitt \& Crowder, 1992).

A particularly instructive demonstration of this is owing to Seither-Preisler et al. (2007), whose experiment has some design similarities with London's study. Just as London included rhythm pairs in which the rhythm with the slower nominal tactus tempo had a faster "surface rate of motion," SeitherPreisler et al. presented pairs of tones in which the tone with the lower pitch had a brighter timbre. They achieved this by relying on the "missing fundamental" phenomenon, whereby a virtual pitch corresponding to a physically absent fundamental frequency is perceived on the basis of the spacing of higher harmonics. By spacing high-frequency harmonics more closely, a lower pitch percept could be brought about than in another tone whose lower-frequency harmonics were spaced more widely. However, these authors found that only musically trained participants relied on this virtual pitch when judging which of two tones was higher. Nonmusicians relied on timbre instead, and amateur musicians gave in-between results. The magnitude of the pitch difference played a role as well.

I argued above that the subjective tactus of a rhythm is somewhat analogous to the virtual pitch of a complex tone (especially that of a Shepard tone), and its subdivisions are analogous to harmonics. A rhythm with a fast surface rate of motion thus has a "brighter timbre," as it were, than a rhythm with a slow surface rate. It seems not implausible that nonmusicians, especially, might confuse this "brighter timbre" with a "higher pitch" (faster tempo). Just as a complex tone has higher-frequency components that could be mistaken for the virtual pitch of the tone (and indeed these partials have a high pitch when considered in isolation or "heard out" from the complex), a fast rhythm has high-tempo components that could be mistaken for its virtual tempo, the tactus. In Seither-Preisler's words, the "perceptual focus" of musicians is on the fundamental, whereas that of nonmusicians is on the spectral composition of the sound. Similarly, musicians' perceptual focus might be on the tactus tempo, whereas nonmusicians might be swayed easily by faster-moving metrical levels. However, London used participants with a wide range of musical experience and did not find significant effects of that variable, so perhaps the analogy has its limits. 


\section{CONCLUSIONS}

By suggesting that London's participants relied on subjective tactus tempi in making their judgments, I have been expressing doubts about London's conclusions that "our perception of musical speed or tempo is more than simple apprehension of the tactus rate" (p. 1) and that "focusing on the tactus level does not improve the overall accuracy and consistency of tempo judgments" (p. 10, boldface in original). In the first statement, London means the nominal tactus, but my point is that participants probably relied on their subjective tactus instead. The second statement refers to the subjective tactus, however, and thus expresses a truism if judgments are based on the subjective tactus in the first place. Instructions to focus on the nominal tactus might have yielded quite different results. The notion of a "musical speed" different from the subjective tactus seems unnecessary to me, though tactus ambiguity and misperception of the tempo of a chosen tactus are possible. I do agree completely with London's conclusion that "the manner/rate of sensorimotor coordination one has with a rhythmic pattern may affect one's sense of its speed" (p. 10). His participants probably tapped (or imagined tapping) with their subjective tactus, which is a form of sensorimotor coordination, and made their tempo judgments accordingly. ${ }^{[3]}$

\section{NOTES}

[1] I am leaving aside here potential expressive fluctuations in tempo, which complicate tempo measurement and judgment (see, e.g., Repp, 1994) but are not relevant to London's study, which used strictly isochronous rhythms.

[2] At least it has no global tempo. Local tempo can always be defined as the inverse of event inter-onset intervals and may be constant over short time spans.

[3] Note added in proof: The interested reader is encouraged also to look up two highly relevant studies I failed to cite in this commentary: McKinney and Moelants (2006) and Madison and Paulin (2010). The former supports the stance I have been taking, whereas the latter pursues the more controversial notion of "musical speed."

\section{REFERENCES}

Epstein, D. (1995). Shaping time: Music, the brain, and performance. New York: Schirmer.

London, J. (2011). Tactus $\neq$ tempo: Some dissociations between attentional focus, motor behavior, and tempo judgment. Empirical Musicology Review, Vol. 6, pp. 43-55.

Madison, G. (2009). An auditory illusion of infinite tempo change based on multiple temporal levels. PLoS ONE, Vol. 4, e8151.

Madison, G., \& Paulin, J. (2010). Ratings of speed in real music as a function of both original and manipulated beat tempo. Journal of the Acoustical Society of America, Vol. 128, pp. 3032-3040.

McKinney, M. F., \& Moelants, D. (2006). Ambiguity in tempo perception: What draws listeners to different metrical levels? Music Perception, Vol. 24, pp. 155-166.

Parncutt, R. (1994). A perceptual model of pulse salience and metrical accent in musical rhythms. Music Perception, Vol. 11, pp. 409-464.

Pitt, M. A. (1994). Perception of pitch and timbre by musically trained and untrained listeners. Journal of Experimental Psychology: Human Perception and Performance, Vol. 20, pp. 976-986. 
Pitt, M. A., \& Crowder, R. G. (1992). The role of spectral and dynamic cues in imagery for musical timbre. Journal of Experimental Psychology: Human Perception and Performance, Vol. 18, pp. 728-738.

Quinn, S., \& Watt, R. (2006). The perception of tempo in music. Perception, Vol. 35, pp. 267-280.

Repp, B. H. (1994). On determining the basic tempo of an expressive music performance. Psychology of Music, Vol. 22, pp. 157-167.

Repp, B. H. (2008). Metrical subdivision results in subjective slowing of the beat. Music Perception, Vol. 26, pp. 19-39.

Repp, B. H., \& Bruttomesso, M. (2009). A filled duration illusion in music: Effects of metrical subdivision on the perception and production of beat tempo. Advances in Cognitive Psychology, Vol. 5, pp. 114-134.

Repp, B. H., \& Thompson, J. M. (2010). Context sensitivity and invariance in perception of octaveambiguous tones. Psychological Research, Vol. 74, pp. 437-456.

Seither-Preisler, A., Krumbholz, K., Patterson, R., Johnson, L., Nobbe, A., Seither, S., \& Lütkenhöner, B. (2007). Tone sequences with conflicting fundamental pitch and timbre changes are heard differently by musicians and nonmusicians. Journal of Experimental Psychology: Human Perception and Performance, Vol. 33, pp. 743-751.

Shepard, R. (1964). Circularity in judgments of relative pitch. Journal of the Acoustical Society of America, Vol. 36, pp. 2346-2353.

Terhardt, E., Stoll, G., Schermbach, R., \& Parncutt, R. (1986). Tonhöhenmehrdeutigkeit, Tonverwandtschaft und Identifikation von Sukzessivintervallen [Pitch ambiguity, pitch relatedness, and identification of successive intervals]. Acoustics, Vol. 61, pp. 57-66.

van Noorden, L., \& Moelants, D. (1999). Resonance in the perception of musical pulse. Journal of New Music Research, Vol. 28, pp. 43-66. 